J. Electroanal. Chem., 319 (1991) 101-110

Elsevier Sequoia S.A., Lausanne

JEC 01755

\title{
Fractal characterisation of electrodispersed gold electrodes
}

P. Ocon a , P. Herrasti a ${ }^{\text {, L. Vázquez }}{ }^{\text {b }}$, R.C. Salvarezza ${ }^{1, a}$, J.M. Vara ${ }^{\text {a }}$ and A.J. Arvia ${ }^{c}$

${ }^{a}$ Departamento de Química Física Aplicada, C-II, Universidad Autónoma de Madrid, 28049 Madrid (Spain)

${ }^{b}$ Instituto de Ciencias de Materiales, Sede B CSIC Departamento de Física Aplicada, C-XII, Consejo Superior de Investigaciones Cientificas, Universidad Autónoma de Madrid, 28049 Madrid (Spain)

' INIFTA, Universidad Nacional de La Plata, Sucursal 4, Casilla de Correo 16, 1900 La Plata (Argentina)

(Received 14 June 1991)

\begin{abstract}
The fractal dimension of the surface, $D_{\mathrm{s}}$, of thin columnar gold electrodeposits (surface roughness factor 50-100) grown on gold wire cathodes by electroreducing hydrous gold oxide layers has been determined by measuring the diffusion controlled current of the $\mathrm{Fe}(\mathrm{CN})_{6}^{4-} / \mathrm{Fe}(\mathrm{CN})_{6}^{3-}$ reaction. The diffusion current $(I)$ vs. time $(t)$ relationships obeyed a $I \alpha t^{-\alpha}$ dependence with $D_{\mathrm{s}}=2 \alpha+1$. The initial $D_{\mathrm{s}}$ value is $2.5 \pm 0.1$, and decreases to $2.3 \pm 0.1$ by keeping the deposit in contact with the electrolyte solution at $298 \mathrm{~K}$ due to the smoothing of the rough metal surface by surface diffusion.
\end{abstract}

\section{INTRODUCTION}

Surface disorder and random roughness can play a determining role in many physical and chemical properties of surfaces and interfaces [1]. Highly dispersed metals used in heterogeneous catalysis and electrocatalysis exhibit extremely rough and irregular surfaces which can be characterised by using fractal geometry [2]. The demonstration of fractal geometry and the determination of the fractal dimension for real systems is difficult as the fractal behaviour does not extend over all lengths and the experimental methods are also limited to a certain range of scale lengths [2]. As the structure of thin metal films comprises nanometre-sized particles, a method based on scanning tunnelling microscopy (STM) has been recently developed to distinguish the fractal nature of solid materials in the

\footnotetext{
$\overline{1}$ Permanent address: INIFTA, La Plata, Argentina.
} 
nanometre range [3]. This method was used to characterise the surface of thin columnar gold films grown from the electroreduction of oxide layers accumulated on gold wire cathodes [4] and to determine the fractal dimension of the specimen surface, $D_{\mathrm{s}}$ [5].

In this paper a macroscopic method was employed to estimate the fractal dimension of the gold electrodeposits based on the diffusion to a fractal surface [6]. The present method confirms the fractal nature of the deposit surface and the influence of the slow reorganisation processes at the surface of the deposit immersed in the electrolyte solution on the value of $D_{s}$. A good correlation between the present results and those recently reported by using the STM method is obtained.

\section{EXPERIMENTAL}

Columnar structured gold deposits were prepared by electroreducing hydrous gold oxide (hereafter denoted as gold oxide) grown on polycrystalline gold wire electrodes (length $=0.2 \mathrm{~cm}$, radius $=0.05 \mathrm{~cm}$ ) placed in a conventional three-compartment electrochemical cell containing $0.5 \mathrm{M} \mathrm{H}_{2} \mathrm{SO}_{4}$ at $298 \mathrm{~K}$ [4]. The gold oxide layer was accumulated by anodising the gold electrode at $2.6 \mathrm{~V}$ vs. the standard hydrogen electrode (SHE) for a time $t_{\mathrm{a}}$. By changing $t_{\mathrm{a}}$, the amount of the gold oxide can be varied conveniently so that films with average thicknesses ranging between 10 and $1000 \mathrm{~nm}$ can be produced. Subsequently, the gold oxide layer was electroreduced by applying a potential scan from $1.64 \mathrm{~V}$ to $0.34 \mathrm{~V}$ at 0.02 $\mathrm{V} \mathrm{s}^{-1}$. Under these experimental conditions, a columnar-type gold electrodeposit is grown on the gold substrate [7]. The surface area and the roughness factor, $R$, of these deposits was evaluated through voltammetry by using the O-electrodesorption charge and taking the O-electrodesorption monolayer charge density equal to $0.420 \mathrm{mC} \mathrm{cm}^{-2}$ for a polycrystalline gold electrode [8].

Slow reorganisation processes at the rough columnar gold films were detected by keeping them during $10^{5} \mathrm{~s}$ at open circuit in $0.5 \mathrm{M} \mathrm{H}_{2} \mathrm{SO}_{4}$ solution at $298 \mathrm{~K}$. For these specimens, a decrease in the electrodeposit area can be observed, i.e. the value of the surface area changed from $8 \mathrm{~cm}^{2}$ to $1 \mathrm{~cm}^{2}$ after $10^{4} \mathrm{~s}[7,9]$. This effect is due to the increase in grain size in order to decrease the surface Gibbs energy of the entire system [9].

Conventional voltammetry and potentiostatic current transient measurements in the diffusion-controlled regime were performed in a three-compartment electrochemical cell. The electrolyte solution was $5 \mathrm{mM} \mathrm{K}_{4}\left[\mathrm{Fe}(\mathrm{CN})_{6}\right]+5 \mathrm{mM} \mathrm{K}_{3}\left[\mathrm{Fe}(\mathrm{CN})_{6}\right]$ in $0.5 \mathrm{M} \mathrm{NaNO}_{3}$ [10]. Occasionally $10 \mathrm{mM} \mathrm{NaCN}$ was also added to the electrolyte solution to check the influence of the $\mathrm{CN}^{-}$ion, but in this concentration range the latter did not cause any effect on the current transients. The latter were run by stepping the potential from $0.5 \mathrm{~V}$ to $1.1 \mathrm{~V}$ (SHE) and recording the current with a Tektronix digital oscilloscope.

STM measurements were made using a piezo tube STM microscope operating in air. Pt tips cut from a $0.2 \mathrm{~mm}$ diameter wire were used. STM measurements 
were run at 1-2 nA constant tunnel current, and $0.1 \mathrm{~V}$ tunnel voltage (tip positive). The STM data were acquired with a fully automated workstation and stored as binary files with, typically, $256 \times 256$ pixels. STM imaging was complemented with conventional scanning electron microscope (SEM) observations.

RESULTS

Let us first consider the structure of gold electrodeposits resulting from STM and SEM imaging. The STM gray scale topographies of recently prepared gold electrodeposits obtained at different magnifications are shown in Fig. 1. Small rounded grains with sizes ranging between 10 and $20 \mathrm{~nm}$ are clearly observed at
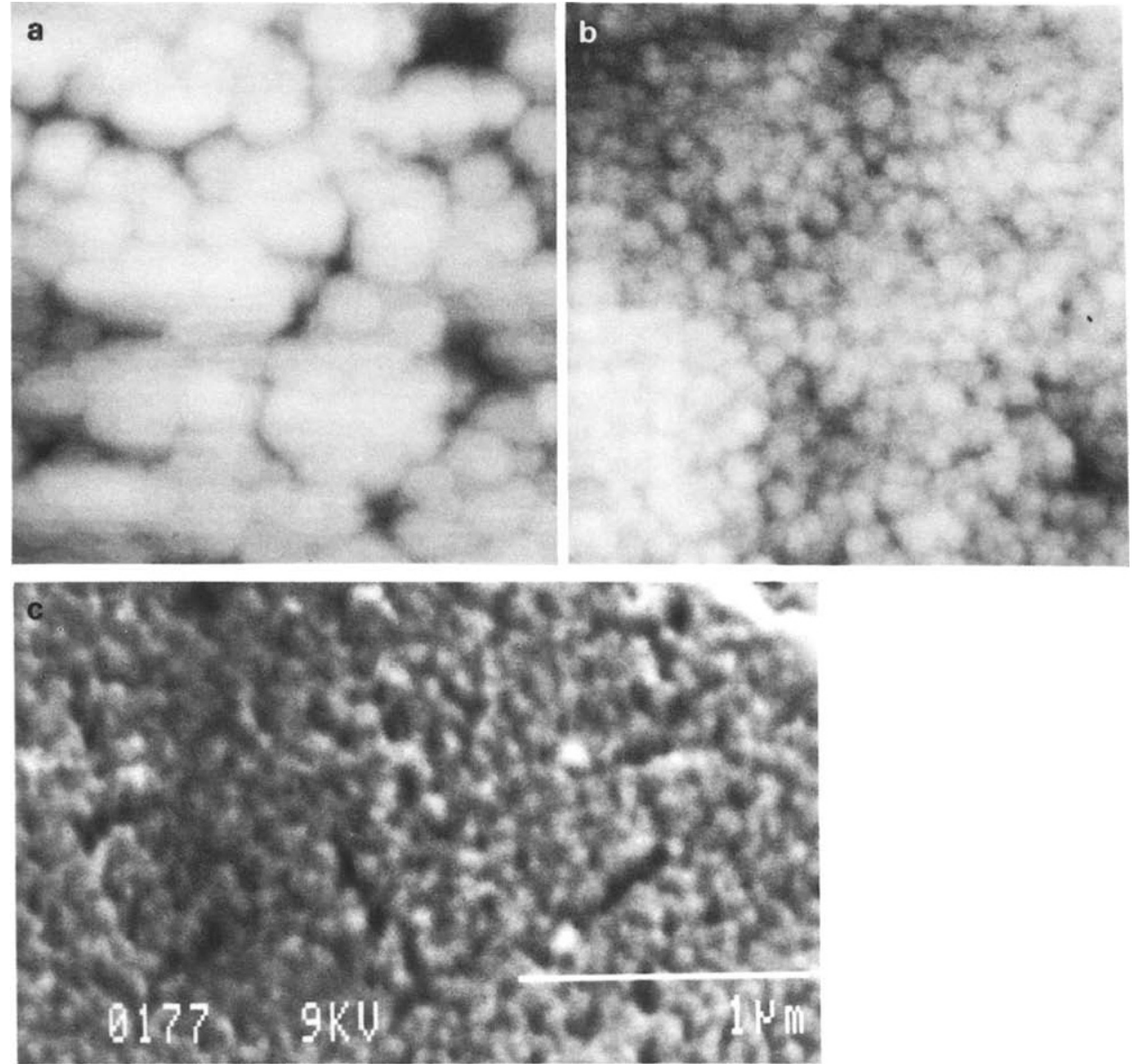

Fig. 1. STM images of a gold electrodeposit $(R=50)$; (a) $0.3 \times 0.3 \mu \mathrm{m}$; (b) $1 \times 1 \mu \mathrm{m}$. (c) SEM micrograph of the same gold electrodeposit. The bar indicates $1 \mu \mathrm{m}$. 


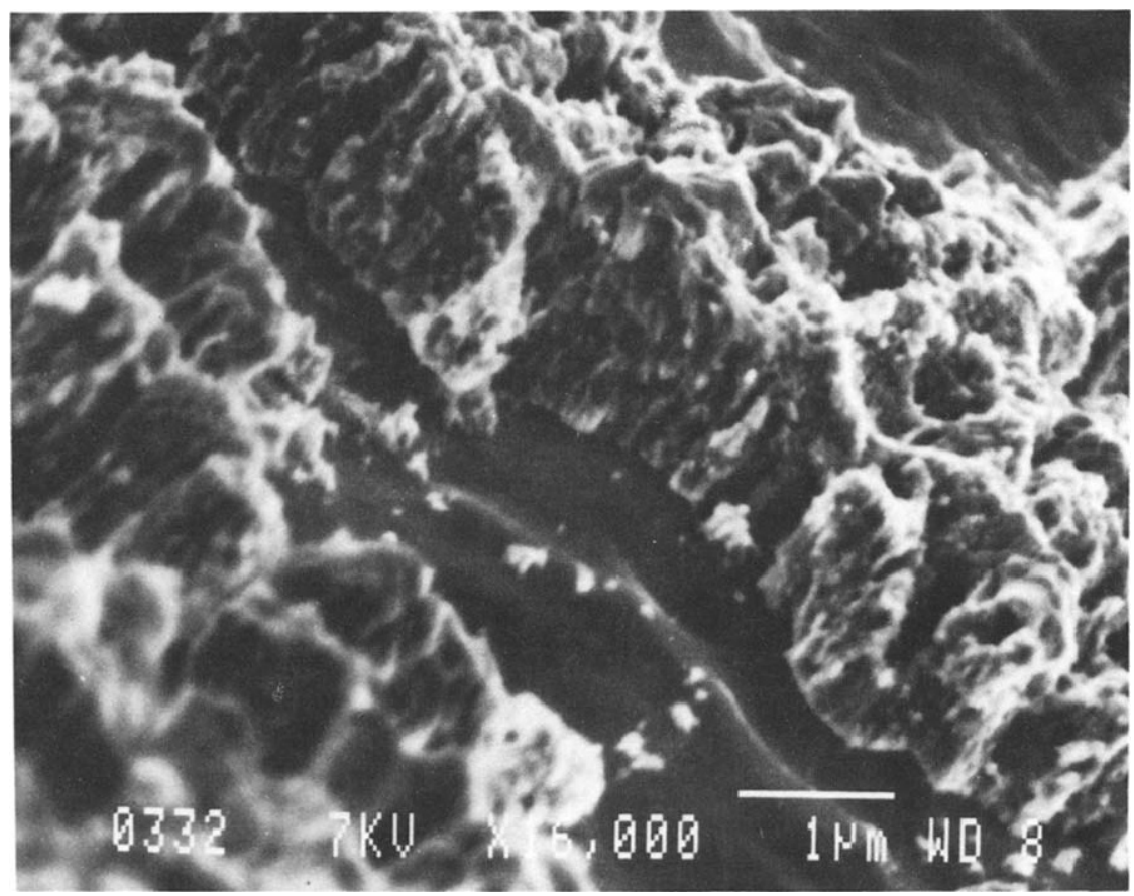

Fig. 2. SEM micrograph of the cross-section of a gold electrodeposit $(R=80)$. The bar indicates $1 \mu \mathrm{m}$.

the higher resolutions (Fig. 1a). These grains, which correspond to the top of the columnar elements of the film, cannot be resolved clearly by SEM (Fig. 1c). However, ramified self-similar voids are observed at all length scales, indicating the fractal nature of the gold electrodeposit surfaces. The cross-section of the electrodeposits (Fig. 2) shows that the film itself is constituted by elongated grains with sizes ranging between 500 and $3000 \mathrm{~nm}$. These features are consistent with a columnar-like structure [7]. The latter are better described as a self-affine rather than a self-similar fractal [11,12]. It should be noted that the intersection of a plane with a self-affine surface generates self-similar lakes or islands $[5,13]$.

Typical voltammograms run at $0.01 \mathrm{~V} \mathrm{~s}^{-1}$ for a smooth gold wire $(R=1.1)$ and for a gold electrodeposit $\left(t_{\mathrm{a}}=2 \mathrm{~min}, R=50\right)$ exhibit the pair of conjugated current peaks and the diffusional limiting currents related to the $\left[\mathrm{Fe}(\mathrm{CN})_{6}\right]^{4-}-\left[\mathrm{Fe}(\mathrm{CN})_{6}\right]^{3-}$ redox couple (Fig. 3). The diffusional current is similar at both the smooth and the rough electrode surfaces, indicating that the microscopic structure of the rough electrode is not seen by the electroactive species. For the rough electrode the voltammogram becomes broader [14] as the scan rate increases from $0.01 \mathrm{~V} \mathrm{~s}^{-1}$ to $0.1 \mathrm{~V} \mathrm{~s}^{-1}$.

Diffusion-controlled current transients for the $\left[\mathrm{Fe}(\mathrm{CN})_{6}\right]^{4-} \Rightarrow\left[\mathrm{Fe}(\mathrm{CN})_{6}\right]^{3-}+\mathrm{e}^{-}$ reaction (Fig. 4a) were obtained by employing a smooth gold wire $(R=1.1)$, a gold electrodeposit grown on the gold wire $(R=50)$, and the same electrodeposit after 


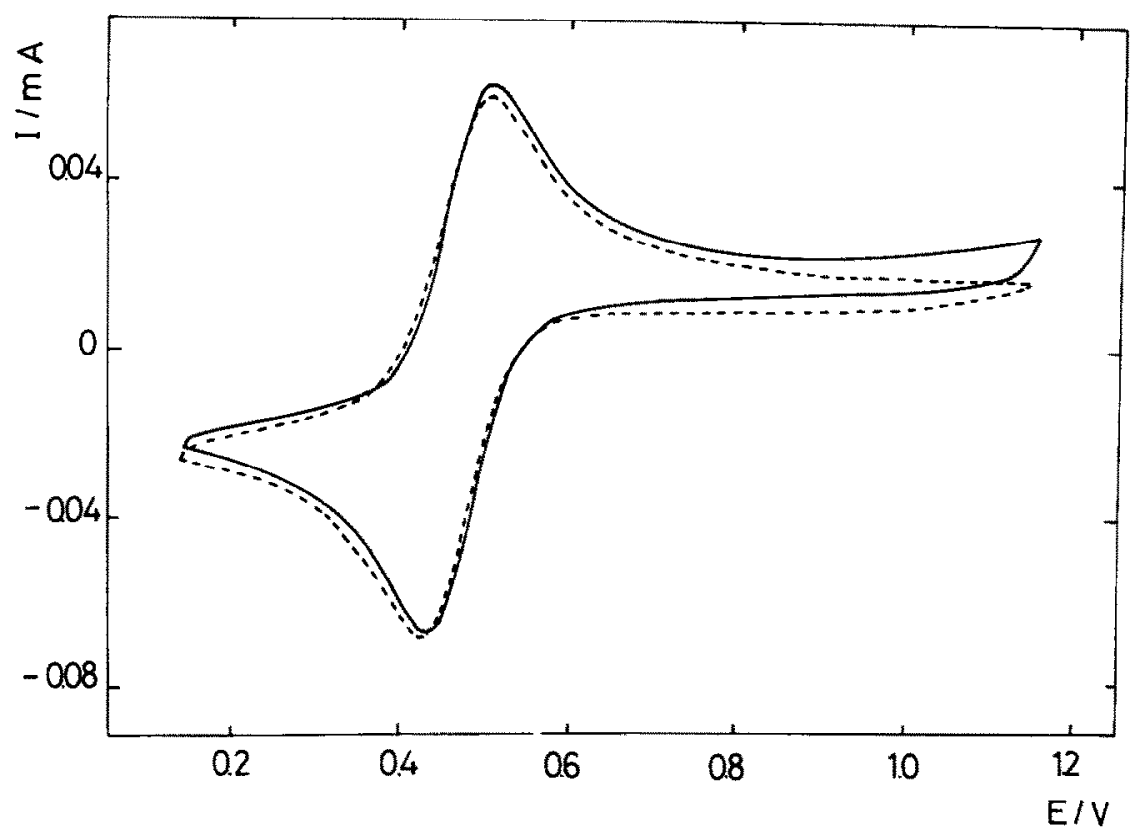

Fig. 3. Voltammograms of the $\left[\mathrm{Fe}(\mathrm{CN})_{6}\right]^{4-} /\left[\mathrm{Fe}(\mathrm{CN})_{6}\right]^{3-}$ redox couple on gold electrodes in the base solution at $0.01 \mathrm{~V} \mathrm{~s}^{-1}$. Dashed line, $R=1.1$, and full line $R=50$.

it has been "aged" at open circuit in $0.5 \mathrm{M} \mathrm{H}_{2} \mathrm{SO}_{4}$ for $2 \times 10^{5} \mathrm{~s}(R=3)$. These results which are displayed as $\log I$ vs. $\log t$ plots (Fig. 4a) exhibit for the smooth gold wire a linear response within the time scale of the experiments with a slope $\alpha=+0.50 \pm 0.05$. On the other hand, the same plots for the recently prepared and for the "aged" gold electrodeposits show a linear behaviour with $\alpha=0.72 \pm 0.05$ and $0.61 \pm 0.05$, respectively, extending from $t=4 \times 10^{-3} \mathrm{~s}$ to $t_{\mathrm{c}}=0.04 \mathrm{~s}$. The value of $t_{\mathrm{c}}$ is the time from which the experimental points deviate from the slope $\alpha$.

Similar experiments made using a gold electrodeposit with $R=100\left(t_{\mathrm{a}}=5 \mathrm{~min}\right)$ and after keeping the same electrodeposit in the electrolyte for $t=8 \times 10^{4} \mathrm{~s}$ $(R=20)$ and for $t=2 \times 10^{5} \mathrm{~s}(R=20)$ yield $\alpha=0.76 \pm 0.05, \alpha=0.62 \pm 0.05$ and $\alpha=0.66 \pm 0.05$, respectively (Fig. $4 \mathrm{~b}$ ). This means that $\alpha$ remains constant for $t>1 \times 10^{5} \mathrm{~s}$. Besides, for these rough electrodeposits, the deviations observed in the linear $\log I$ vs. $\log t$ plots move towards large $t$ values.

\section{DISCUSSION}

For a non-stationary electrochemical reaction under diffusion control the diffusion layer thickness increases with time and the concentration gradient for the diffusing species decreases. When the process involves a soluble reacting species in solution diffusing under semi-infinite linear conditions towards a planar electrode, 

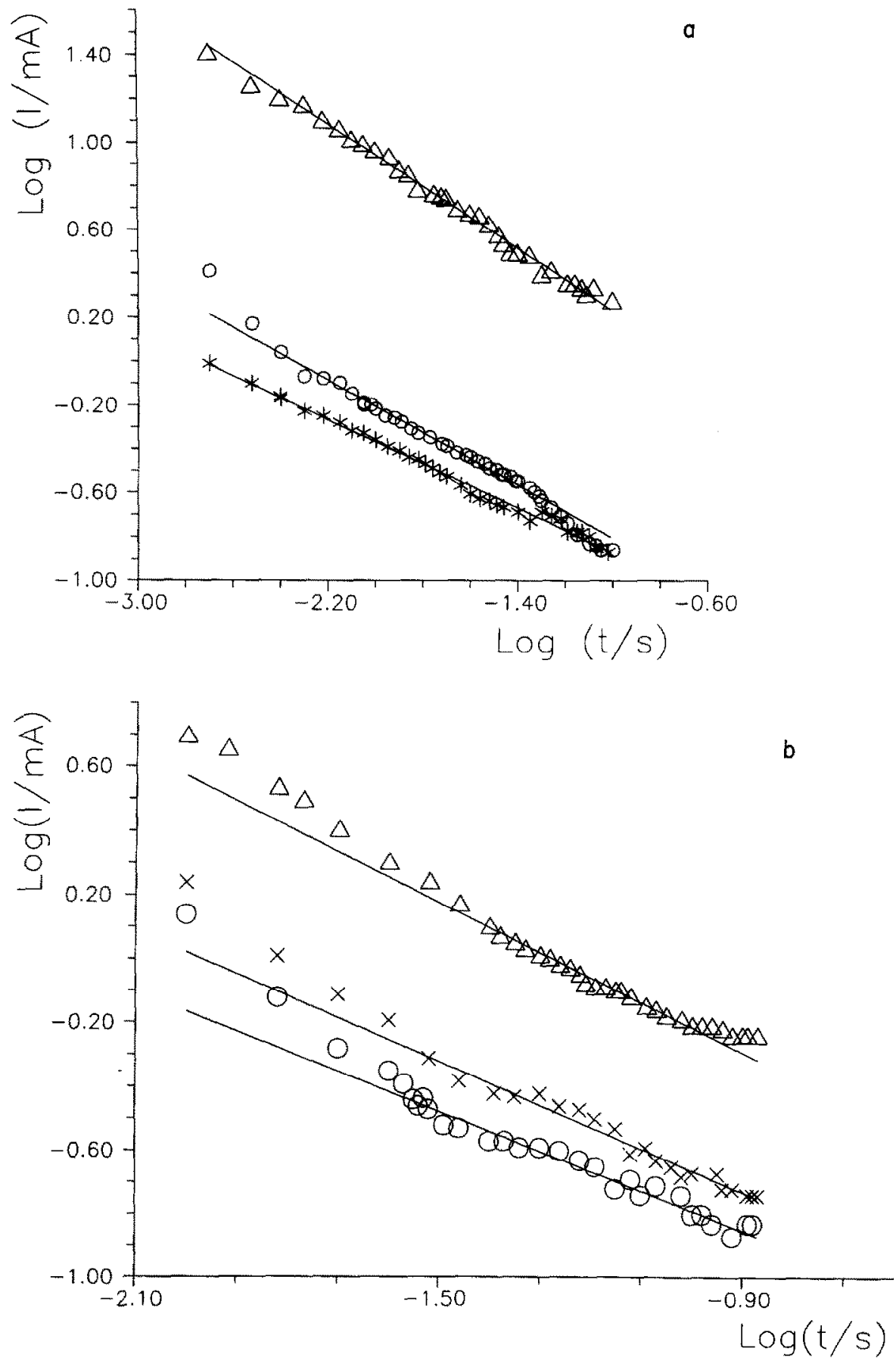
the non-stationary diffusion-controlled current is given by the Cottrell equation [10]:

$I=n F c(D / \pi t)^{0.5}$

where $c$ and $D$ are the bulk concentration and the diffusion coefficient of the electroactive species, and $n$ and $F$ have the usual meaning.

A different situation arises for the case of diffusion towards fractal surfaces. This problem has been studied extensively from both theoretical and experimental viewpoints [6,15]. In this case, the time dependence of the diffusion current becomes more complex, involving a $I \propto t^{-\alpha}$ relationship for $t<t_{\mathrm{c}}$ and the fulfilment of eqn. (1), i.e. $I \propto t^{-0.5}$, for $t>t_{\mathrm{c}}$. The value of $t_{\mathrm{c}}$ is related to the size of the largest irregularities, $\lambda$, at rough surfaces by [15]:

$\lambda^{2} \cong D t_{\mathrm{c}}$

where $D$ is the diffusion coefficient of the electroactive species. From eqn. (2), taking $D=5 \times 10^{-6} \mathrm{~cm}^{2} \mathrm{~s}^{-1}$ (estimated from the slope of a $I$ vs. $t^{-1 / 2}$ linear plot for the smooth gold wire), and $\lambda \cong 3 \times 10^{-4} \mathrm{~cm}$ (Fig. 2) the $I$ vs. $t^{-\alpha}$ behaviour is expected for $t<0.03 \mathrm{~s}$. The relation between $\alpha$ and the fractal dimension of the electrode surface, $D_{\mathrm{s}}$, is given by $[10,15]$ :

$D_{\mathrm{s}}=2 \alpha+1$

From eqn. (3) and the $\alpha$ values shown in Fig. 4 one obtains $D_{\mathrm{s}}=2.0 \pm 0.1$ for the smooth gold wire, $D_{\mathrm{s}}=2.5 \pm 0.1$ for the gold electrodeposits and $D_{\mathrm{s}}=2.3 \pm 0.1$ for electrodeposits which have been "aged" in contact with the electrolyte. The values of $D_{\mathrm{s}}$ are practically independent of $R$ within \pm 0.1 , at least when $R$ is changed between 50 and 100 . The difference between the values of $D_{\mathrm{s}}$ of recently prepared electrodeposits and those obtained for the same electrodes after "ageing" in the electrolyte is related to the slow reorganisation phenomena occurring at the metal surface [7,9]. In the present case the slow reorganisation process can be followed through the decrease in the $D_{\mathrm{s}}$ value up to about $10^{5} \mathrm{~s}$. For $t>10^{5} \mathrm{~s}$, the $D_{\mathrm{s}}$ value remains close to $2.3 \pm 0.1$. It should be noted that for a cauliflower platinum electrode the diffusion-controlled current measurements give $D_{\mathrm{s}}=2.46[10]$ in good agreement with the $D_{\mathrm{s}}$ value obtained in the present work for recently prepared gold electrodes. For platinum electrodeposits $D_{\mathrm{s}}$ is practically time independent [3] as its surface diffusion coefficient in the electrolyte at $25^{\circ} \mathrm{C}$ is lower than that reported for gold [9].

Fig. 4. (a) $\log I$ vs. $\log t$ for different gold electrodes: ( $*$ ) smooth gold wire $(R=1.1), \alpha=0.50 \pm 0.05$; $(\Delta)$ gold electrodeposit $(R=50), \alpha=0.72 \pm 0.05$; $(O)$ gold electrodeposit after "ageing" in $0.5 \mathrm{M}$ $\mathrm{H}_{2} \mathrm{SO}_{4}$ for $t=10^{5} \mathrm{~s}(R=3), \alpha=0.61 \pm 0.05$. (b) $\mathrm{Log} I$ vs. $\log t$ for different gold electrodes: $(\Delta)$ gold electrodeposit $(R=100), \alpha=0.76 \pm 0.05$; $(O)$ gold electrodeposit after "ageing" in $0.5 \mathrm{M} \mathrm{H}_{2} \mathrm{SO}_{4}$ for $t=8 \times 10^{4} \mathrm{~s}(R=20), \alpha=0.62+0.05 ;(\times)$ gold electrodeposit after "ageing" in $0.5 \mathrm{M} \mathrm{H}_{2} \mathrm{SO}_{4}$ for $t=2 \times 10^{5} \mathrm{~s}(R=20), \alpha=0.66 \pm 0.05$. 
TABLE 1

Values of $D_{\mathrm{s}}$ for different electrodeposits

\begin{tabular}{lll}
\hline & $\begin{array}{l}\text { Diffusion-controlled reaction } \\
\text { measurements } \\
D_{\mathrm{s}}\end{array}$ & $\begin{array}{l}\text { STM method } \\
\text { (ref. 3) } \\
D_{\mathrm{s}}\end{array}$ \\
\hline $\begin{array}{l}\text { Gold electrodeposits } \\
R=50\end{array}$ & 2.4 & - \\
$R=80$ & - & 2.5 \\
$R=100$ & 2.5 & \\
"Aged" gold electrodeposits & \\
$R=3$ & 2.2 & - \\
$R=10$ & - & 2.3 \\
$R=20$ & $2.2-2.0$ & - \\
Platinum electrodeposits & 2.46 (ref. 10) & \\
$R=?$ & - & 2.5 \\
$R=80$ & - & 2.5 \\
"Aged" platinum electrodeposits & \\
$R=68$ & - & \\
\hline
\end{tabular}

The values of $D_{\mathrm{s}}$ derived for gold electrodeposits from the diffusion-controlled current transients agree with those recently determined by the ex-situ STM technique [3] (Table 1). This coincidence is particularly significant in justifying some assumptions implied in the STM method used to obtain the fractal dimension of rough surfaces. In this method $D_{\mathrm{s}}$ is estimated from $D_{\mathrm{p}}$, the fractal dimension of the perimeter of either lakes or islands generated by the intersection
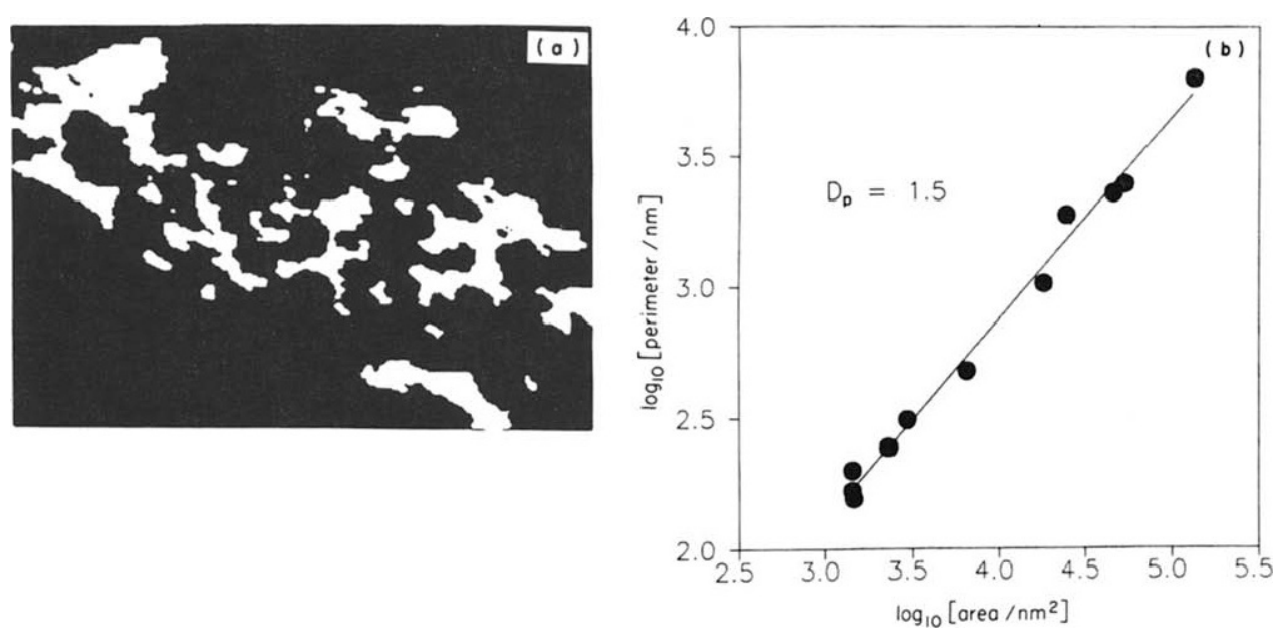

Fig. 5. (a) Pattern of lakes generated by STM image processing for a gold electrodeposit $(R=80)$. (b) $\log P$ vs. $\log A$ plot for the pattern depicted in (a). The value of $D_{\mathrm{p}}$ resulting from (b) is 1.5 . From ref. 3. 
of a plane with the fractal surface. A plot of $\log P$ (perimeter) vs. $\log A$ (area) for the lakes or islands is made to obtain the value of $D_{\mathrm{p}}[5,13]$ (Fig. 5). The latter is related to $D_{\mathrm{s}}$ by the following equation:

$D_{\mathrm{s}}=D_{\mathrm{p}}+1$

although there are exceptions to eqn. (4) [5]. Besides, as the STM provides information at the nanometre scale, a large number of STM images has to be processed to obtain a representative $D_{\mathrm{s}}$ value. Therefore, the present results allow us to conclude that both methods are equally efficient for the determination of the fractal dimension of electrodeposited metal surfaces such as gold and platinum (Table 1).

Finally, it should be noted that $D_{\mathrm{s}}=2.5$ would be consistent with a random columnar growth process controlled by electric or concentration fields [2].

\section{CONCLUSIONS}

The fractal dimension of the surface of gold electrodeposits has been determined from diffusion-controlled current transient measurements of the $\left[\mathrm{Fe}(\mathrm{CN})_{6}\right]^{4-} /\left[\mathrm{Fe}(\mathrm{CN})_{6}\right]^{3-}$ reaction. The results confirm the fractal nature of the surface of gold electrodeposits grown far from the equilibrium conditions. The influence of the surface diffusion of metal atoms producing a smoothing of the surface reflects in the decrease of the value of the fractal dimension of the surfaces in contact with the electrolyte solution. The present results also demonstrate the validity of the assumption made in the determination of the fractal dimension of rough metal surfaces by STM image processing.

\section{ACKNOWLEDGEMENT}

A fellowship from the Comisión Interministerial de Ciencia y Tecnología (Spain) and the Consejo Nacional de Investigaciones Científicas y Técnicas (Argentina) to R.C.S. is gratefully acknowledged. Financial support for this work was obtained from project MAT89-0204 CICYT (Spain).

\section{REFERENCES}

1 G.A. Somorjai, Chemistry in Two Dimensions: Surfaces, Cornell University Press, Ithaca, NY, 1981.

2 P. Meakin in D. Avnir (Ed.), The Fractal Approach to Heterogeneous Chemistry, John Wiley \& Sons, New York, 1989.

3 J.M. Gómez-Rodriguez, L. Vázquez, A.M. Baró, C. Alonso, R.C. Salvarezza, J.M. Vara and A.J. Arvia, J. Phys. Chem., in press.

4 A. Chialvo, W.E. Triaca and A.J. Arvia, J. Electroanal. Chem., 171 (1984) 303.

5 B.B. Mandelbrot, The Fractal Geometry of Nature, Freeman, San Francisco, CA, 1977.

$6 \mathrm{~S}$. Havlin in D. Avnir (Ed.), The Fractal Approach to Heterogeneous Chemistry, John Wiley \& Sons, New York, 1989. 
7 L. Vázquez, A. Bartolomé, A.M. Baró, C. Alonso, R.C. Salvarezza and A.J. Arvia, Surf. Sci., 215 (1989) 171.

8 S. Trasatti and O.A. Petrii, Real Surface Area Measurements in Electrochemistry, J. Pure Appl. Chem., 67 (1991) 711.

9 C. Alonso, R.C. Salvarezza, J.M. Vara, A.J. Arvia, L. Vázquez, A. Bartolomé and A.M. Baró, J. Electrochem. Soc., 137 (1990) 2161.

10 T. Pajkossy, J. Electroanal. Chem., 30 (1991) 1.

11 P. Meakin, J. Phys. A, 20 (1987) L1113.

12 A. Family and T. Vicsek, J. Phys. A, 18 (1985) L75.

13 J. Feder, Fractals, Plenum Press, New York, 1988.

14 T. Pajkossy and L. Nyikos, Electrochim. Acta, 34 (1989) 181.

15 T. Pajkossy and L. Nyikos, Electrochim. Acta, 34 (1989) 171. 